\title{
La precipitación está asociada con la fenología de Rodriguezia granadensis (Lindl.) Rchb. f. en Fusagasugá, Cundinamarca
}

\section{Precipitation is associated with phenology of Rodriguezia granadensis (Lindl.) Rchb. f. in Fusagasugá, Cundinamarca}

\author{
Arlette Ivonne Gil Clavijo; María Alejandra Bohórquez²; Yenny Yohana Reyes ${ }^{3}$
}

1 Ingeniera Agrónoma, M.Sc. Ciencias Agrarias. Docente investigadora grupo PROSAFIS. Universidad de Cundinamarca. Fusagasugá - Cundinamarca, Colombia; e-mail: aigil@ucundinamarca.edu.co; arlettegil@hotmail.com; (D) http://orcid.org/0000-0001-6536-5877

²Estudiante de Ingeniería Agronómica. Universidad de Cundinamarca, Programa de Ingeniería Agronómica, semillero de investigación PROCREDEVE. Fusagasugá - Cundinamarca, Colombia; e-mail: malejandrabohorquez@ucundinamarca.edu.co; (D) http://orcid.org/0000-0003-2258-0694

${ }^{3}$ Estudiante de Ingeniería Agronómica. Universidad de Cundinamarca, Programa de Ingeniería Agronómica, semillero de investigación PROCREDEVE. Fusagasugá - Cundinamarca, Colombia; e-mail: yyohanareyes@ucundinamarca.edu.co; (D) http://orcid.org/0000-0002-1447-6435

Cómo citar: Gil Clavijo, A.G.; Bohórquez, M.A.; Reyes, Y.Y. 2020. La precipitación está asociada con la fenología de Rodriguezia granadensis (Lindl.) Rchb. f. en Fusagasugá, Cundinamarca. Rev. U.D.C.A Act. \& Div. Cient. 23(1):e1511. http://doi.org/10.31910/rudca. v23.n1.2020.1511

Artículo de acceso abierto publicado por Revista U.D.C.A Actualidad \& Divulgación Científica, bajo una licencia Creative Commons CC BY-NC 4.0

Publicación oficial de la Universidad de Ciencias Aplicadas y Ambientales U.D.C.A, Institución de Educación Superior Acreditada de Alta Calidad por el Ministerio de Educación Nacional.

Recibido: Febrero 1 de 2020

Aceptado Junio 3 de 2020

Editado por: Ingeborg Zenner de Polanía

\section{INTRODUCCIÓN}

La fenología, se refiere a los cambios visibles en el desarrollo biológico y se explica en términos de "estadios" o "fenofases" (Meier, 1997; Tang et al. 2016). Los estudios que documentan la fenología de las especies epífitas son relativamente pocos, debido a la complejidad para el seguimiento de las plantas que viven en el dosel de los árboles (Sheldon \& Nadkarni, 2015).

Rodriguezia granadensis (Lindl.) Rchb. f. es una orquídea epífita, que se encuentra distribuida en Colombia, en el rango altitudinal entre 700 a $1.900 \mathrm{~m}$ s.n.m., es común que florezca entre marzo a septiembre en los bosques andinos (Calderón-Sáenz, 2006), aunque Gil \& Jácome (2014), la reportan en un rango de 1.800 a $3.900 \mathrm{~m}$ s.n.m., para el departamento de Cundinamarca.
Con el fin de estudiar el efecto de la precipitación sobre las fenofases de la orquídea nativa Rodriguezia granadensis (Lindl.) Rchb. f., se realizó esta investigación, en el entorno de la hacienda Betania, municipio de Fusagasugá, debido a la escasa información sobre este tópico en particular. La toma de datos, se efectuó entre septiembre de 2018 y junio de 2019, monitoreando mensualmente la fenología en cuatro parcelas, tabulando los individuos de R. granadensis, en los estadios vegetativo, floración (flor abierta) y cápsula.

En las orquídeas, el inicio de la fenofase de floración, se ha relacionado directamente con los incrementos de la precipitación, mientras que la fructificación está asociada a temporadas secas, debido a que la mayoría de las especies son anemocóricas (LemusJiménez \& Ramírez, 2002; Vieira-Faria et al. 2007; Duarte et al. 2017). 
Actualmente, las investigaciones sobre la relación fenológica y la precipitación son escasas en Colombia, para esta orquídea nativa, por lo que este estudio aportará a este conocimiento y contribuirá con información básica. Aunque se encuentre catalogada en la categoría de riesgo de preocupación menor (Calderón-Sáenz, 2006) es importante entender los cambios registrados en su ciclo de vida y según Barman \& Devadas (2013), se debe proveer un seguimiento a los procesos que promuevan el mantenimiento, la restauración y la conservación de orquídeas.

\section{MATERIALES Y MÉTODOS}

Esta investigación, se llevó a cabo en la hacienda Betania, municipio de Fusagasugá (Cundinamarca) ubicada a 04² $21^{\prime} 37^{\prime \prime} \mathrm{N}, 74^{\circ} 22^{\prime} 29^{\prime}$ 'W, con altitud de $1.765 \mathrm{~m}$ s.n.m., temperatura media anual de $20^{\circ} \mathrm{C}$ y precipitación media anual superior a los $1.200 \mathrm{~mm}$. La toma de datos correspondió al lapso comprendido entre septiembre de 2018 a junio de 2019. Se monitoreó mensualmente la fenología de las plantas de $R$. granadensis y se relacionó con la precipitación, medida con una estación meteorológica marca Davis Vantage Vue 6250 (Davis Instruments Corp. Inc., Hayward, California, U.S.A.).

La hacienda Betania cuenta con un área total de 7,74ha y, a partir de varios recorridos de reconocimiento, se llevó a cabo un conteo de individuos de R. granadensis, que se encontraban creciendo sobre árboles de guayaba (Psidium guajava L.), como forofitos. A partir de este conocimiento, se demarcaron cuatro parcelas, con un área de $250 \mathrm{~m}^{2}$ cada una, ubicadas al Occidente, al Norte y al Oriente del predio.

Basados en la metodología de Ordóñez-Blanco \& Parrado-Roselli (2017), se tomaron 40 plantas al azar dentro de cada parcela, desde el nivel del suelo hasta una altura de $2 \mathrm{~m}$, con el fin de minimizar el efecto del microclima, teniendo, de esta manera, un modelo estadístico completamente al azar.

Para la determinación de las fenofases, se siguió el modelo desarrollado por Bravo-Monasterio et al. (2012), tabulando cada individuo, según su desarrollo, como $\mathrm{V}$ (vegetativo), F (floración) y C (cápsula). Para categorizar la duración de las fenofases de floración y cápsula (fructificación), se usaron los rangos sugeridos por Newstrom et al. (1994), donde, si el período es menor a 1 mes, se considera breve, entre 1 y 5 meses es categorizada como intermedia y extendida, si es mayor a 5 meses. Para analizar la relación entre la precipitación y la fenología, se realizó un análisis de correlación por rangos de Spearman, determinando la asociación o interdependencia entre las variables, mediante el programa estadístico InfoStat versión 2017 (Di Rienzo et al. 2017).

\section{RESULTADOS Y DISCUSIÓN}

La especie $R$ granadensis ha sido descrita como una orquídea epífita de ramita, que presenta polimorfismo para el color de las flores: blanco y rosa. Las flores de las plantas en la hacienda Betania presentaron en su totalidad un color blanco (Figura 1), mientras que Ospina-Calderón et al. (2013; 2015), en sus estudios sobre las poblaciones presentes de esta orquídea en la Reserva Forestal Bosque de Yotoco (Valle del Cauca, Colombia), mencionan también flores de la variación rosada.

Las plantas de R. granadensis, se encontraron creciendo sobre árboles de guayaba (P. guajava L.), como forofitos, que concuerda con los resultados obtenidos en la investigación de VentreLespiaucq et al. (2017), realizada en un parque natural de bosque tropical andino y de Arévalo et al. (2011), en las epífitas de ramita Hirtzia escobarii y Rodriguezia lehmannii, en Cundinamarca y Risaralda,

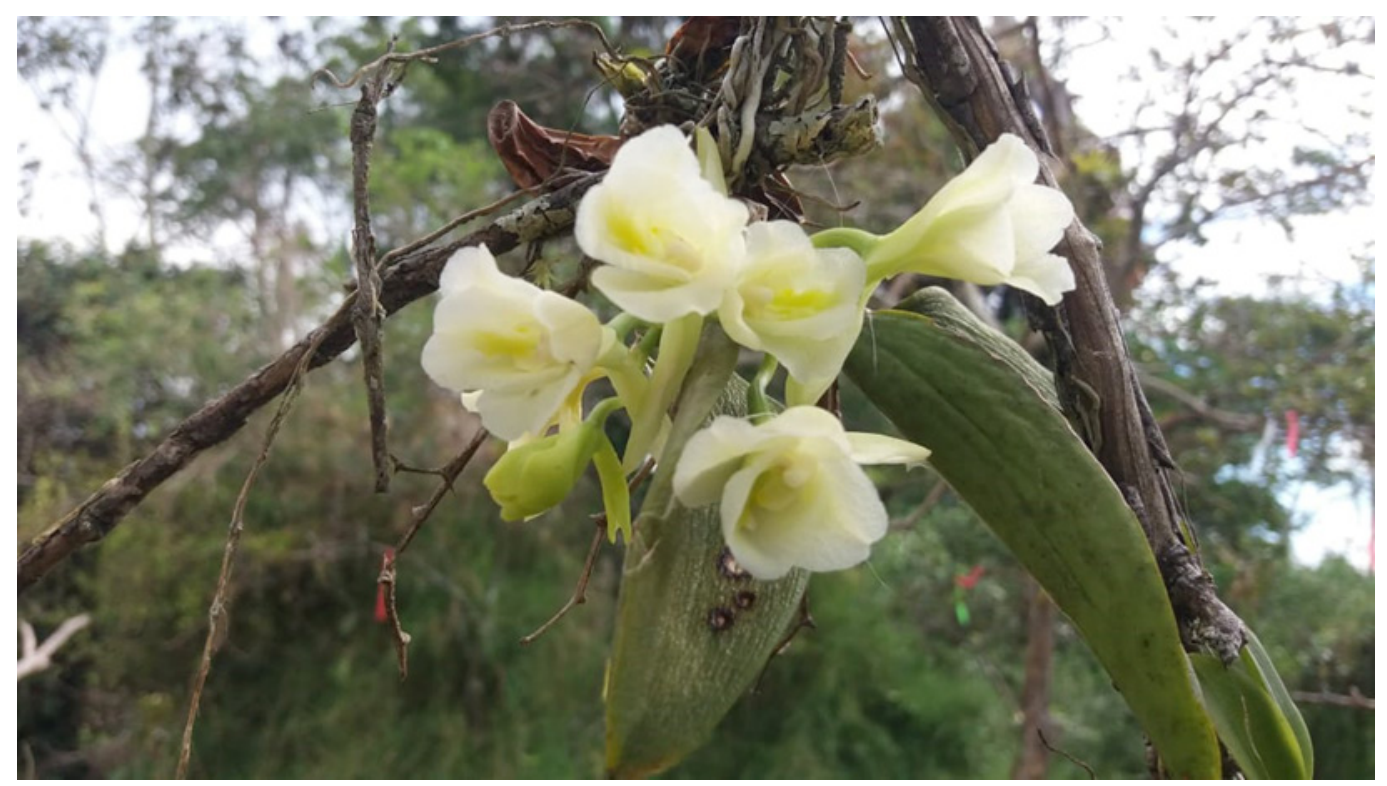

Figura 1. Rodriguezia granadensis, fenotipo de flores blancas, en el sitio del estudio. 
Colombia, respectivamente. Según Granados-Sánchez et al. (2003), las orquídeas epífitas dependen de la niebla, el rocío y la lluvia y sus raíces se extienden afuera del forofito hacia la atmósfera, donde el velamen de la raíz se carga de agua después de la lluvia y en tiempo seco, se llena de aire y actúa como aislante.

Los resultados mostraron que la fenofase vegetativa para el número de individuos registrados predominó durante todo el lapso evaluado, presentando una leve disminución en los períodos lluviosos y un aumento en la época seca (Figura 2), obteniendo una correlación negativa (Spearman, $\mathrm{p}<0,05)$ con la precipitación $(\mathrm{r}=-0,79)$, lo que indicaría la disminución del número de individuos vegetativos, cuando se presentan las épocas de mayor precipitación en la zona de la hacienda Betania. Este resultado contrasta con el reportado por Ticktin et al. (2016), en la bromelia Tillandsia macdongallii L. B. Sm. (epífita), en Oaxaca, México, donde la estación seca hizo que las plantas fueran más sensibles a la desecación, aumentando su tasa de mortalidad.

En las épocas de mayor precipitación (octubre-noviembre de 2018 y abril-mayo de 2019), con máximos de 171 y 138mm de lluvia, respectivamente, se incrementó el número de plantas en floración (Figura 2). Los períodos coinciden con lo reportado en el Valle del Cauca, por Ospina-Calderón et al. (2015), quienes también afirman que la mayoría de las orquídeas epífitas sincronizan su fase reproductiva con los patrones de lluvia.

Sahagún-Godínez (1996) indica que la floración de 54 géneros de orquídeas, incluido Rodriguezia, en el oeste de México, estuvo fuertemente influenciada por la lluvia, con un pico en la época de mayor precipitación y que el significado ecológico de la apertura floral ocurre para sincronizar su floración. Texier et al. (2018) afirman que la mayoría de los taxones de orquídeas en África occidental producen flores al inicio y durante la mayor parte de la estación lluviosa. Los ejemplos anteriores muestran que se requiere un mínimo nivel de humedad para la floración y que la precipitación juega un papel fundamental en la inducción floral.

Para la fase fenológica de cápsula, la tendencia observada fue de un incremento en el número de individuos para las épocas de mayor precipitación, en octubre-noviembre de 2018 y abril-mayo de 2019 (Figura 2). Resultados obtenidos por Fan et al. (2012), en el sureste de China, para la orquídea epífita Acampe rigida (Buch.-Ham. Ex Sm.) P. F. Hunt, mostraron que la lluvia promueve la polinización y, por lo tanto, contribuye de manera sustancial en el incremento del cuajado de las cápsulas. Según Pezzini et al. (2008), la maduración de los frutos se ve favorecida por períodos secos y la baja humedad relativa del aire contribuye con la desecación y, finalmente, con la dispersión de las semillas anemócoras. Las semillas de las orquídeas son diminutas y sin reservas nutricionales, por lo que su dispersión durante los períodos secos podrían impedir su germinación y posterior establecimiento (Baskin \& Baskin, 2001).

La prueba de correlación por rangos de Spearman $(\mathrm{p}<0,05)$ para las fenofases de floración y de cápsula de R. granadensis presentó una correlación positiva fuerte con la precipitación $(\mathrm{r}=0,75 \mathrm{y}$ $\mathrm{r}=0,65$, respectivamente), indicando el incremento del número de individuos en estas fases, como respuesta al aumento de las lluvias. Estos resultados pueden tener su base en que R. granadensis requiere de alta humedad para ingresar a la floración y posterior formación de cápsula, como mecanismos de éxito reproductivo.

En cuanto a la duración de los periodos de floración y de cápsula (fructificación) de R. granadensiBr, se obtuvo que fueron intermedios,

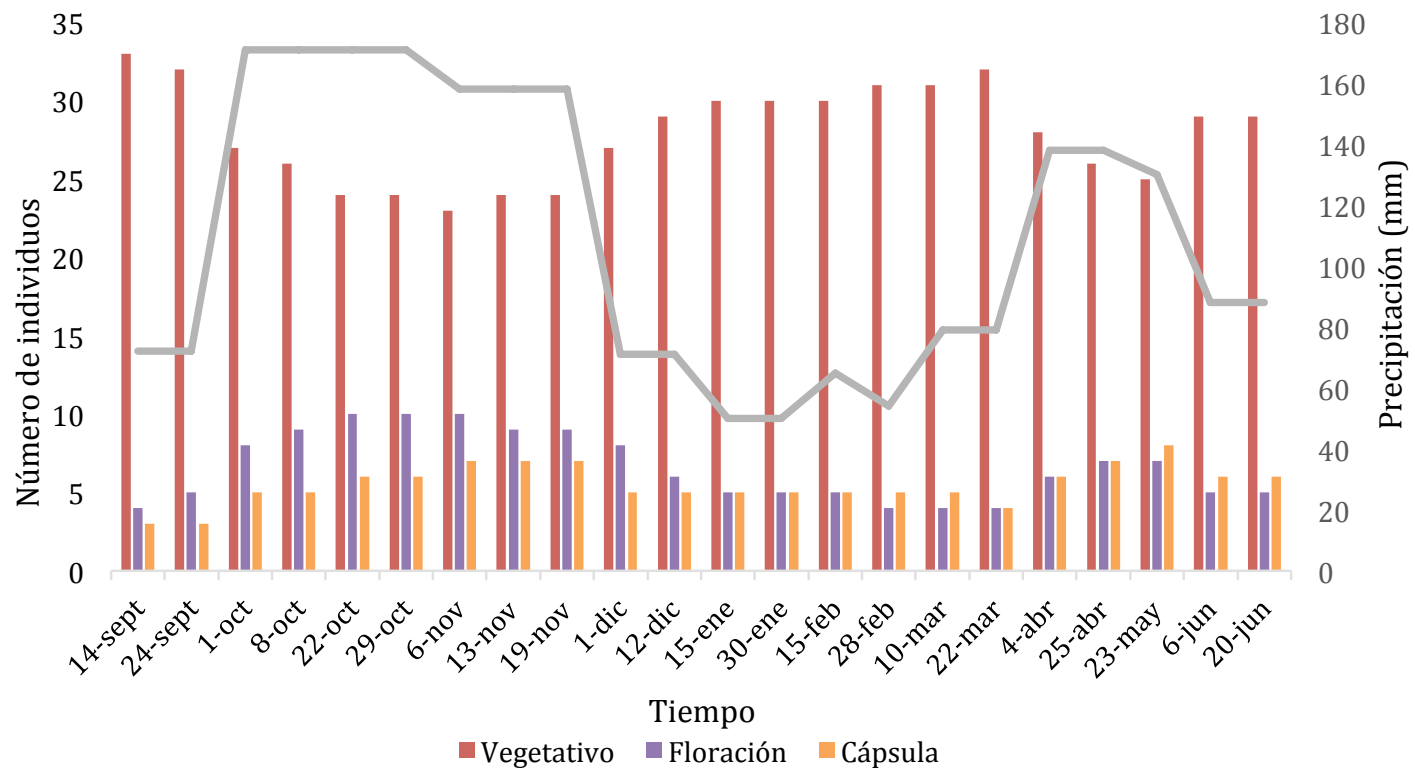

Figura 2. Número de individuos por fase de desarrollo de Rodriguezia granadensis en respuesta a la precipitación, Fusagasugá-Cundinamarca. 
con una duración entre 1 a 5 meses, según la clasificación de Newstrom et al. (1994), que concuerda con los resultados obtenidos en la investigación de Reina-Rodríguez \& Otero (2011), para esta especie, en el valle del río Cauca.

Para concluir, se puede inferir que las respuestas fenológicas de las plantas son adaptativas, bajo las condiciones climáticas cambiantes. Cabe resaltar que esta investigación fue corta, por lo que se recomienda ampliar el lapso, pero a manera de información primigenia en el área de influencia de la zona de estudio, se constituye en un valioso aporte, como base para futuros estudios, a fin de entender la ecofisiología y la apropiación de mecanismos, para su conservación in situ.

Agradecimientos: A la Oficina de Turismo y a la Secretaría de Desarrollo Económico y Competitividad del municipio de Fusagasugá, por permitir el desarrollo de esta investigación. A César Ariza, por su apoyo con el análisis estadístico. Conflictos de intereses: El manuscrito fue preparado y revisado con la participación de todos los autores, quienes declaramos que no existe ningún conflicto de intereses que ponga en riesgo la validez de los resultados presentados.

\section{REFERENCIAS}

1. ARÉVALO, R.; FIGUEROA, J.; MADRIÑÁN, S. 2011. Anatomía foliar de ocho especies de orquídeas epífitas. Lankesteriana. 11(1):39-54. https://doi.org/10.15517/ LANK.V11I1.18314

2. BARMAN, D.; DEVADAS, R. 2013. Climate change on orchid population and conservation strategies: a review. J. Crop and Weed. 9(2):1-12.

3. BASKIN, C.C.; BASKIN, J.M. 2001. Seeds: Ecology, Biogeography and Evolution of Dormancy, and Germination. Academic Press, San Diego. 666p.

4. BRAVO-MONASTERIO, P.; SAN MARTÍN, J.; BAEZA-H., G. 2012. Distribución, abundancia y fenología de orquídeas en un bosque caducifolio endémico de Chile central. Polibotánica. 33:117-129.

5. CALDERÓN-SÁENZ, E. (ed.). 2006. Libro Rojo de Plantas de Colombia. Volumen 6: Orquídeas, Primera Parte. Serie Libros Rojos de Especies Amenazadas de Colombia. Bogotá, Colombia. Instituto Alexander von Humboldt - Ministerio de Ambiente, Vivienda y Desarrollo Territorial. $828 \mathrm{p}$.

6. DI RIENZO, J.A.; CASANOVES, F.; BALZARINI, M.G.; GONZÁLEZ, L.; TABLADA, M.; ROBLEDO, C.W. 2017. InfoStat versión 2017. Grupo InfoStat, FCA: Universidad Nacional de Córdoba, Argentina.

7. DUARTE, E.; MANGEÓN, V.; KÜPPERS, G.; ROCHA, S.; NIELLA, F. 2017. Tamaño y viabilidad de semillas: implicancias en la evolución y conservación de Phaius tankervilleae (Orchidaceae). Caldasia, 39(2):388-399. https://doi.org/10.15446/caldasia.v39n2.62184

8. FAN, X.; BARRET'T, S.; LIN, H.; LINGLING, C.; ZHOU, X.; LIU, Q. 2012. Rain pollination provides reproductive assurance in a deceptive orchid. Annals of Botany. https://doi.org/110.953-8.10.1093/aob/mcs165

9. GIL, K.S.; JÁCOME, J. 2014. Representatividad de las colecciones "ex situ" de la familia Orchidaceae en la franja altitudinal entre bosque andino y páramo, en el departamento de Cundinamarca, Colombia. Orquideología. 31(2):144-172.

10. GRANADOS-SÁNCHEZ, D.; LÓPEZ-RÍOS, G.F.; HERNÁNDEZ-GARCÍA，M.A.; SÁNCHEZGONZÁLEZ, A. 2003. Ecología de las plantas epífitas. Revista Chapingo. Serie Ciencias Forestales y del Ambiente. 9(2):101-111

11. LEMUS-JIMÉNEZ, L.J.; RAMÍREZ, N. 2002. Fenología reproductiva en tres tipos de vegetación de la planicie costera de la península de Paraguaná, Venezuela. Acta Científica Venezolana. 53(4):266-278.

12. MEIER, U. 1997. BBCH-Monograph, Growth Stages of Plants. Berlin: Blackwell Wissenschafts-Verlag.

13. NEWSTROM, L.E.; FRANKIE, G.W.; BAKER, H.G. 1994. A new classification for plant phenology based on flowering patterns in lowland tropical rain forest trees at La Selva, Costa Rica. Biotropica. 26:141-159.

14. ORDÓÑEZ-BLANCO, J.C.; PARRADO-ROSSELLI, A. 2017. Relación fenología-clima de cuatro especies de orquídeas en un bosque altoandino de Colombia. Lankesteriana. 17(1):1-15. http://dx.doi.org/10.15517/ lank.v17i1.27897

15. OSPINA-CALDERÓN, N.H.; DUQUE-BUITRAGO, C.A.; TREMBLAY, R.L.; OTERO, J.T. 2015. Pollination ecology of Rodriguezia granadensis (Orchidaceae). Lankesteriana. 15(2):129-139. https://doi. org/10.15517/LANK.V15I2.20745

16. OSPINA-CALDERÓN, N.H.; OTERO, J.T.; TREMBLAY, R.L. 2013. Selección de rasgos florales en Rodriguezia granadensis (Lindl.) Rchb.f. (Orchidaceae): estudio de la eficacia biológica en una especie polimórfica. Proc. fourth scientific conference on andean orchids. Guayaquil, Ecuador, October 31-November 04, 2012. Resúmenes. Lankesteriana. 13(1-2):144-145. SANTO, M.M.; JACOBI, C.M.; FERNANDES, G.W. 
2008. Polinizacão, dispersão de sementes e fenologia de espécies arbóreas no Parque Estadual da Mata Seca. MG Biota. 1:37-45.

18. REINA-RODRÍGUEZ, G.A.; OTERO, J.T. 2011. Guía ilustrada de las orquídeas del valle geográfico del río Cauca y piedemonte andino bajo. Santiago de Cali: Asociación Vallecaucana de Orquideología-Universidad Nacional de Colombia. (Sede Palmira). 95p.

19. SAHAGÚN-GODÍNEZ, E. 1996. Trends in the phenology of flowering in the Orchidaceae of western Mexico. Biotropica. 28(1):130-136. https://doi. $\operatorname{org} / 10.2307 / 2388778$

20. SHELDON, K.S.; NADKARNI, N.M. 2015. Reproductive phenology of epiphytes in Monteverde, Costa Rica. Revista de Biología Tropical, 63(4):1119-1126.

21. TANG, J.; KÖRNER, C.; MURAOKA, H.; PIAO, S.; SHEN, M.; THACKERAY, S.J.; YANG, X. 2016. Emerging opportunities and challenges in phenology: a review. Ecosphere. 7(8):e01436. https://doi.org/10.1002/ ecs 2.1436

22. TEXIER, N.; DEBLAUWE, V.; STÉVART, T.; SONKÉ, B.; SIMO-DROISSART, M.; AZANDI, L.; BOSE,
R.; DJUIKOUO, M.N.; KAMDEM, G.; KAMDEM, N.; MAYOGO, S.; ZEMAGHO, L.; DROISSART, V. 2018. Spatio-temporal patterns of orchids flowering in Cameroonian rainforests. Int. J. Biometeorol. 62:19311944. https://doi.org/10.1007/s00484-018-1594-3

23. TICKTIN, T.; MONDRAGÓN, D.; GAOUE, O.G. 2016. Host genus and rainfall drive the population dynamics of a vascular epiphyte. Ecosphere. 7(11):e01580. http://doi. org/10.1002/ecs2.1580

24. VENTRE-LESPIAUCQ, B.A.; DELGADO, J.A.; OSPINACALDERÓN, N.H.; OTERO, J.T.; ESCUDERO, A.; SÁNCHEZ, M.A.; BALAGUER, L.; FLANAGAN, N.S. 2017. A tropical epiphytic orchid uses a low-light interception strategy in a spatially heterogeneous light environment. Biotropica. 49(3):318-327. https://doi. org/10.1111/btp.12425

25. VIEIRA-FARIA, M.; ANDRADE, M.; BITTENCOURT, N.; CARVALHO, O.R. 2007. Flowering phenology, nectary structure and breeding system in Corymborkis fava (Spiranthoideae: Tropidieae), a terrestrial orchid from a Neotropical forest. Australian J. of Botany. 55:635-642. 